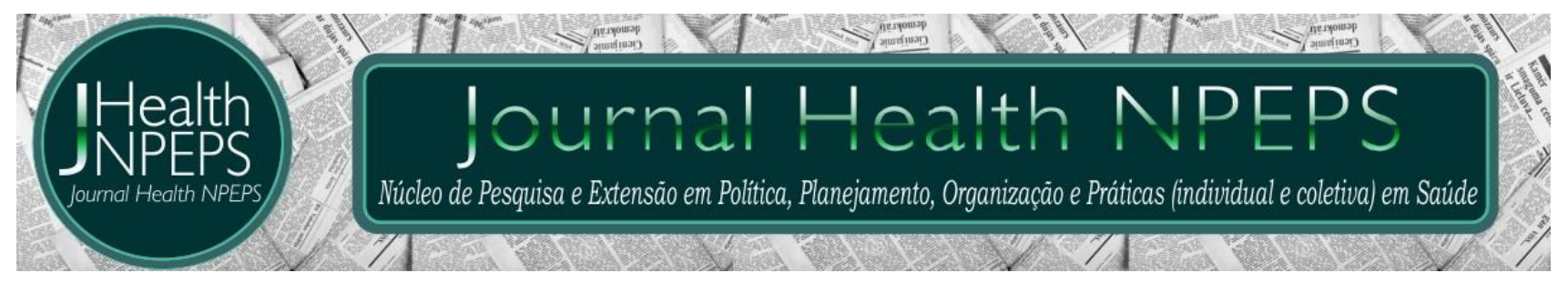

http://dx.doi.org/10.30681/252610105177

ARTIGO ORIGINAL

\title{
Actitud ante el consumo de alcohol en adolescentes del Noreste de México*
}

Attitude towards alcohol consumption in adolescent from Northeast Mexico

Atitude em relação ao consumo de álcool em adolescentes do Nordeste do México

\begin{abstract}
Roberto Ontiveros-Patiño ${ }^{1}$, Gustavo Gutiérrez Sánchez ${ }^{2}$, Ma de la Luz Martínez Aguilar ${ }^{3}$, Hermelinda Ávila-Alpirez ${ }^{4}$, Jesús Alejandro Guerra-Ordoñez ${ }^{5}$, Tirso Duran-Badillo ${ }^{6}$
\end{abstract}

\section{RESUMEN}

Objetivo: describir la actitud ante el consumo de alcohol y la relación con la edad, grado escolar y género de los estudiantes de una preparatoria del Noreste de México. Método: diseño descriptivo y correlacional, en una población de 208 estudiantes de preparatoria. Se aplicó el instrumento ACTICOL-92 con alpha de Cronbach de .87. Resultados: se encontró puntuación promedio en las actitudes de 81.00 (DE=8.12), actitud preventiva de 32.07 ( $D E=5.53)$, actitud permisiva de 12.26 ( $D E=4.04)$, consecuencias negativas de 3.50 (DE=3.50), facilitación social de 7.64 (DE=2.38) y actitud evasiva de 8.92. La actitud preventiva, actitud permisiva y facilitación social son diferentes en mujeres en comparación con hombres $(p<.05)$. A mayor edad mayor actitud permisiva y mayor percepción de consecuencias negativas y a mayor edad menor actitud evasiva $(p<.05)$. Conclusión: la actitud preventiva, permisiva, evasiva, percepción de consecuencias negativas y facilitación social son alta en los estudiantes.

\footnotetext{
*Artículo parte de la disertación de maestria en psicología clónica y educativa "Actitud de los estudiantes de preparatoria ante el consumo de alcohol", presentada en la Unidad Académica Multidisciplinaria Matamoros - Universidad Autónoma de Tamaulipas, Matamoros, Tamaulipas, México.
}

${ }^{1}$ Licenciado en Psicología. Maestro en Psicología Clínica y Educativa. Unidad Académica Multidisciplinaria Matamoros - Universidad Autónoma de Tamaulipas (UAMM-UAT). Matamoros, Tamaulipas, México. E-mail: ontiveros r@hotmail.com ORCID ID: http://orcid.org/0000-0002-3185-2260

${ }^{2}$ Licenciatura en Psicología. Doctor en Psicología. UAMM-UAT. Matamoros, Tamaulipas, México. E-mail: ggutierrez@docentes.uat.edu.mx ORCID ID: http://orcid.org/0000-0002-5919-3914

${ }^{3}$ Licenciatura en Enfermería. Doctora en Enfermería y Cultura de los Cuidados. UAMM-UAT. Matamoros, Tamaulipas, México. Email: madelaluzmartinez@yahoo.com.mx ORCID ID: http://orcid.org/0000-0001-9003-907X

${ }^{4}$ Licenciatura en Enfermería. Doctora en Enfermería y Cultura de los Cuidados. UAMM-UAT. Matamoros, Tamaulipas, México. Email: avila@docentes.uat.edu.mx ORCID ID: http://orcid.org/0000-0001-5286-5944

${ }^{5}$ Licenciatura en Enfermería. Doctor en Ciencias de Enfermería. UAMM-UAT. Matamoros, Tamaulipas, México. E-mail: jesus.guerra@docentes.uat.edu.mx ORCID ID: http://orcid.org/0000-0002-4587-3214

${ }^{6}$ Licenciatura en Enfermería. Doctor en Metodología de la Enseñanza. UAMM-UAT. Matamoros, Tamaulipas, México. E-mail: tduran@docentes.uat.edu.mx ORCID ID: http://orcid.org/0000-0002-7274-3511 Autor correspondiente: Av. del Maestro SN, Colonia Alianza, Matamoros, Tamaulipas, México. , CP. 87410. 
La actitud preventiva, permisiva y facilitación social son diferentes en mujeres y hombres.

Descriptores: Actitud; Adolescente; Alcoholismo.

\section{ABSTRACT}

Objective: to describe the attitude towards alcohol consumption and the relationship with the age, school grade and gender of the students at a high school in Northeast Mexico. Method: descriptive and correlational design, in a population of 208 high school students. The ACTICOL-92 instrument with Cronbach's alpha of .87 was applied. Results: an average score was found in the attitudes of 81.00 (SD=8.12), preventive attitude of 32.07 (SD=5.53), permissive attitude of 12.26 (SD=4.04), negative consequences of $3.50(S D=3.50)$, social facilitation of $7.64 \quad(S D=2.38)$ and evasive attitude of 8.92. Preventive attitude, permissive attitude and social facilitation are different in women compared to men $(p<.05)$. At older age, greater permissive attitude, and greater perception of negative consequences and at older age, less evasive attitude $(p<.05)$. Conclusions: the preventive, permissive, evasive attitude, perception of negative consequences and social facilitation are high in students. The preventive, permissive and social facilitation attitude is different in women and men.

Descriptores: Attitude; Adolescent; Alcoholism.

\section{RESUMO}

Objetivo: descrever a atitude frente ao consumo de álcool e a relação com a idade, série escolar e sexo de alunos de um colégio do Nordeste do México. Método: desenho descritivo e correlacional, em uma população de 208 alunos do ensino médio. Foi aplicado o instrumento ACTICOL-92 com alfa de Cronbach de 0,87. Resultados: pontuação média em atitudes de $81,00 \quad(\mathrm{DP}=8,12)$, atitude preventiva de 32,07 $(D P=5,53)$, atitude permissiva de $12,26(D P=4,04)$, consequências negativas de 3,50 $(D P=3,50)$, facilitação social de 7,64 (DP=2,38) e atitude de evitação de 8,92. A atitude preventiva, permissiva e facilitação social são diferentes nas mulheres em comparação aos homens $(p<0,05)$. Quanto maior a idade, maior é a atitude permissiva e maior a percepção das consequências negativas. E quanto mais velha é a atitude menos evasiva $(p<0,05)$. Conclusão: a atitude preventiva, permissiva, evasiva, percepção de consequências negativas e facilitação social são elevadas nos estudantes. As atitudes preventivas, permissivas e de facilitação social são diferentes entre mulheres e homens.

Descritores: Atitude; Adolescente; Alcoolismo.

\section{INTRODUCCIÓN}

Las personas inician con el consumo de alcohol por diversas causas, como curiosidad (29.4\%), invitación de amigos (13.5\%), experimentación (12.4 \%), problemas familiares (10\%), influencia de amigos (9.4\%), aceptación del grupo (4.1\%), invitación de familiares (2.9\%) o depresión (2.4\%). En América Latina el primer lugar de consumo de alcohol lo ocupa Chile con el 9.6 litros per cápita, mientras que México ocupa el décimo lugar con un consumo de 7.2 litros per cápita1.

El consumo nocivo de alcohol entre los adolescentes preocupa cada vez más en muchos países, ya que 
reduce el autocontrol y aumenta los comportamientos de riesgo, como las relaciones sexuales no protegidas, violencia (especialmente por parte de la pareja), puede provocar problemas de salud en una etapa posterior de la vida e influir en la esperanza de vida o comportamientos peligrosos en la carretera y muertes prematuras ${ }^{2}$.

La adolescencia es una etapa en donde las personas atraviesan por un conjunto de cambios que aumentan su vulnerabilidad frente a situaciones de riesgo, siendo el consumo de alcohol una de las conductas problemáticas que más se presenta, al consolidarse como una práctica de ocio entre los jóvenes. Se ha evidenciado que el consumo de alcohol en adolescentes es una preocupación social por tener implicaciones económicas, políticas y de salud pública y porque afecta el desarrollo biológico, la salud, los procesos de aprendizaje y la socialización de los jóvenes ${ }^{3}$. Para contribuir en el conocimiento del consumo de alcohol en adolescentes se considera importante describir las actitudes ante el consumo.

El contexto en que los adolescentes se encuentran influye en sus actitudes. En este sentido, se considera que el consumo de alcohol cumple una función social, muchos jóvenes ingresan en el mundo de las sustancias psicoactivas precisamente por este motivo, es una manera de sentir y pensar con base en algunas premisas socioculturales del grupo al que ellos pertenecen. Un aspecto importante para considerar es que la primera ocasión que los adolescentes beben alcohol casi nunca es el resultado de una decisión personal, sino que suele ser la respuesta a una invitación o presión de algunos miembros de la sociedad, los cuales pueden ser familiares. La experiencia por parte del joven suele ser positiva porque le habrá servido para identificarse y ser uno más del grupo, o bien liberarse de la presión social. Este resultado positivo favorece el tránsito de un consumo experimental, a la ingesta frecuente de esta sustancia, especialmente si esta práctica es habitual en el grupo, para posteriormente consolidarse como un hábito o estilo de vida 4 .

Así mismo, el consumo de sustancias se asocia con algunas características de la adolescencia, como la búsqueda de identidad, la autonomía $e$ independencia; así como con la adopción de roles de adulto y la concepción de inmunidad frente a los riesgos, situación que favorece la búsqueda de sensaciones novedosas 
momentáneas e inmediatas. Ante la búsqueda de estas sensaciones novedosas, los adolescentes en ocasiones se involucran en conductas de diferente nivel de riesgo, siendo las de alto riesgo las de mayor preocupación. Dentro de las conductas de riesgo a las cuales se enfrenta el adolescente se encuentran el inicio en la vida sexual sin protección, la adopción de estilos de vida poco saludables, el inicio en el consumo de sustancias a edades mucho más tempranas y el abuso de alcohol, tabaco y otras drogas 5 .

El análisis de las actitudes ante el consumo de alcohol cobra relevancia al relacionarse con la adquisición y apego a estilos de vida que son extendidos e impactan en la etapa adulta. Si las actitudes negativas, evasivas, de facilitación social y permisivas ante el consumo de alcohol son identificadas son identificadas en etapas tempranas, pueden ser redireccionadas, ya que se considera que estas actitudes están condicionadas por las creencias acerca de las consecuencias de la conducta y el valor afectivo que el adolescente atribuye a tales consecuencias ${ }^{6}$.

En la revisión de la literatura, se ha encontrado poca evidencia empírica en la que se aborden las actitudes ante el consumo de alcohol en adolescentes. Por lo que con fundamento en lo ya expuesto y la identificación de necesidad de conocimiento científico acerca de las actitudes de los adolescentes, se consideró importante realizar una investigación que describa la actitud ante el consumo de alcohol y la relación con la edad, grado escolar y género de los estudiantes de una preparatoria del Noreste de México.

\section{MÉTODO}

El estudio fue de tipo descriptivo y correlacional. La población de estudio estuvo conformada por adolescentes de nivel medio superior en una escuela pública de Matamoros, Tamaulipas. En el estudio participaron 208 adolescentes de primero, segundo y tercer año escolar de bachillerato. Se incluyó a todos los adolescentes que aceptaron participar y que contaron con la autorización de su padre o tutor.

Para medir las actitudes hacia el consumo de alcohol, se utilizó la Escala de Actitudes hacia el Consumo de Alcohol, diseñada por Pons y Berjano ${ }^{7}$, evalúa las percepciones y creencias de adolescentes y jóvenes respecto a la percepción de desajuste de la conducta de abuso, permisividad hacia el consumo 
habitual de alcohol, las consecuencias negativas derivadas del consumo, la facilitación social o creencia de que el alcohol es vehículo de socialización y las creencias sobre que el consumo de alcohol reduce la tensión o estrés.

El instrumento está compuesto por 32 items planteados en una escala tipo Likert, sus opciones de respuesta incluyen cuatro posibilidades que son totalmente de acuerdo, bastante de acuerdo, bastante en desacuerdo y totalmente en desacuerdo, el puntaje mínimo es 32 y el máximo es 128, indicando a mayor puntaje mayores son las actitudes hacia el consumo de alcohol. Este instrumento ha sido utilizado y validado en población mexicana encontrando un Coeficiente de Confiabilidad Alpha de Cronbach de $0.87^{8}$.

En la subescala Actitud Preventiva, el adolescente que puntúa alto en este factor está reconociendo en el abuso de alcohol un tipo de conducta socialmente desajustada. En la subescala Actitud Permisiva, una alta puntuación estaría relacionada con el mantenimiento de actitudes indulgentes ante del consumo abusivo de bebidas alcohólicas entre los adolescentes. En cuanto a la subescala Consecuencias Negativas, una puntuación elevada refleja el reconocimiento de que la ingesta continuada de bebidas alcohólicas conduce al riesgo de determinados efectos negativos de índole social y sanitaria. En la subescala Facilitación Social, las puntuaciones altas corresponderían a aquellos adolescentes que perciben el alcohol como un vehículo hacia las relaciones sociales y de la integración grupal. $Y$ finalmente en la subescala Actitud Evasiva, es la percepción del consumo de alcohol basada en la indulgencia y en la minimización de sus consecuencias negativas, pero apoyada sobre todo en la "justificación" aportada por sus efectos evasivos.

La presente investigación se apegó a lo dispuesto en la Ley General de Salud en materia de Investigación en seres humanos 9 . El proyecto fue aprobado por el comité de investigación y ética de la Unidad Académica Multidisciplinaria Matamoros dependiente de la Universidad Autónoma de Tamaulipas (Dictamen 078). Antes de la recolección de datos se solicitó el permiso de la institución educativa, se pidió a los adolescentes que estuvieran de acuerdo en participar en la investigación trajeran firmado el consentimiento por parte de sus padres 
o tutores. Se aplicó el instrumento en el horario de clase.

Los datos fueron procesados y analizados en el paquete estadístico Statistical Package for the Social Sciences (SPSS) versión 21 para Windows. Para describir las características de los sujetos y las actitudes ante el consumo de alcohol se utilizó estadística descriptiva e inferencial, en las que se encuentra frecuencia, porcentaje, medias y desviación estándar.

Se aplicó la prueba de Kolmogorov Smirnov con corrección de Lilliefors $(p=.200)$ y se observó distribución normal en las variables, por lo que se utilizaron las pruebas de
Coeficiente de Correlación de Pearson, $t$ de Student, ANOVA de 1 factor como prueba de comparación de medias y HSD de Tukey como prueba a posteriori.

\section{RESULTADOS}

Los participantes fueron $58.2 \%$ del sexo femenino y edad promedio de 15.88 años (DE=.98). La puntuación promedio obtenida en las actitudes fue de 81.00, en la tabla 1 se encuentran los datos descriptivos de las dimensiones de la actitud ante el consumo de alcohol.

Tabla 1 - Descripción de la actitud ante el consumo de alcohol por dimensiones.

\begin{tabular}{lcccc}
\hline \multirow{2}{*}{ Dimensión } & \multirow{2}{*}{ Media } & \multirow{2}{*}{ DE } & \multicolumn{2}{c}{ IC 95\% } \\
\cline { 4 - 5 } & & & LI & LS \\
\hline Actitudes & 81.00 & 8.122 & 79.72 & 82.21 \\
Actitud preventiva & 32.07 & 5.535 & 31.35 & 32.91 \\
Consecuencias negativas & 20.10 & 3.506 & 19.64 & 20.61 \\
Facilitación social & 7.64 & 2.389 & 7.23 & 7.96 \\
Actitud evasiva & 8.92 & 1.836 & 8.66 & 9.16 \\
\hline
\end{tabular}

En el análisis de las actitudes ante el consumo de alcohol en la escala global no se observaron diferencias estadísticamente significativas por sexo. Sin embargo, se encontraron diferencias significativas en la actitud preventiva $(p=.001)$, actitud permisiva ( $p<.001)$ y facilitación social $(p=.025)$ por sexo; es decir, dichas actitudes son diferentes de acuerdo con el sexo del adolescente (Tabla 2). 
Tabla 2 - Prueba $t$ de Student para las actitudes por sexo. Matamoros, Tamaulipas, México.

\begin{tabular}{|c|c|c|c|c|c|c|c|}
\hline \multirow{2}{*}{ Variables } & \multirow{2}{*}{ Media } & \multirow{2}{*}{$D E$} & \multicolumn{2}{|c|}{ IC 95\% } & \multirow{2}{*}{$t$} & \multirow{2}{*}{ gl } & \multirow{2}{*}{$p$} \\
\hline & & & $\mathrm{LI}$ & LS & & & \\
\hline \multicolumn{8}{|l|}{ Actitudes } \\
\hline Masculino & 81.98 & 8.17 & & & & 206 & \\
\hline Femenino & 83.09 & 8.44 & -3.41 & 1.20 & -.941 & 206 & .348 \\
\hline \multicolumn{8}{|c|}{ Actitud Preventiva } \\
\hline Masculino & 31.21 & 5.70 & -436 & -1.23 & -3.529 & 206 & 001 \\
\hline Femenino & 34.01 & 5.59 & & & & & \\
\hline \multicolumn{8}{|c|}{ Actitud Permisiva } \\
\hline Masculino & 13.51 & 3.97 & 1.06 & 3.23 & 3.916 & 206 & $<.001$ \\
\hline $\begin{array}{c}\text { Femenino } \\
\text { Consecuencia }\end{array}$ & 11.36 & 3.87 & \multicolumn{5}{|c|}{ Consecuencias Negativas } \\
\hline $\begin{array}{l}\text { Masculino } \\
\text { Masecuencias }\end{array}$ & 19.04 & 3.14 & & & & & \\
\hline Femenino & 19.71 & 2.82 & -7.48 & 0.15 & -1.597 & 206 & .112 \\
\hline \multicolumn{8}{|c|}{ Facilitación Social } \\
\hline Masculino & 8.08 & 2.59 & 0.09 & 1.40 & 2.255 & 206 & 025 \\
\hline Femenino & 7.33 & 2.18 & & & & & \\
\hline \multicolumn{8}{|c|}{ Actitud Evasiva } \\
\hline $\begin{array}{l}\text { Masculino } \\
\text { Femenino }\end{array}$ & $\begin{array}{l}8.10 \\
8.19\end{array}$ & $\begin{array}{l}2.16 \\
2.12\end{array}$ & -.68 & .50 & -.288 & 206 & .774 \\
\hline
\end{tabular}

Con la prueba de correlación de Pearson, se identificó una relación positiva y estadísticamente significativa entre la edad y la actitud permisiva $(p<.001)$, consecuencias negativas $(p=.001)$ y relación negativa entre la edad y actitud evasiva $(p<.001)$; es decir, a mayor edad mayor actitud permisiva y mayor percepción de consecuencias negativas, y a mayor edad menor actitud evasiva (Tabla 3).

Referente a las actitudes ante el consumo de alcohol por año escolar, el análisis de varianza de un factor (ANOVA) demuestra la existencia de diferencias estadísticamente significativas en la Actitud Permisiva $(p=.002), \quad$ Consecuencias Negativas $(p<.001)$ y Actitud Evasiva $(p<1.000)$ entre los tres grupos de año escolar (Tabla 4).

Ante la identificación de diferencias significativas entre la actitud permisiva, consecuencias negativas y actitud evasiva por año escolar, se procedió a realizar un análisis de comparaciones múltiples (Prueba pos hoc) a través de la prueba HSD de Tukey, con el objetivo de identificar grupos diferenciados. En este sentido, se observó que no hubo diferencias en la Actitud permisiva ( $p>.05)$ y Actitud evasiva ( $p>.05)$ entre los grupos de primero y segundo. Así mismo, no se encontraron diferencias en las Consecuencias negativas ( $p>.05)$ entre los grupos de segundo y tercero (Tabla 5). 
Tabla 3 - Prueba de Correlación de Pearson entre la edad y actitudes ante el consumo de alcohol. Matamoros, Tamaulipas, México.

\begin{tabular}{llc}
\hline Variables & $r$ & $p$ \\
\hline Actitudes (Global) & .098 & .161 \\
Actitud preventiva & -.050 & .474 \\
Actitud permisiva & .258 & $<.001$ \\
Consecuencias negativas & .239 & .001 \\
Facilitación social & .118 & .088 \\
Actitud evasiva & -.273 & $<.001$ \\
\hline
\end{tabular}

Tabla 4 - Prueba de ANOVA de las actitudes ante el consumo de alcohol por año escolar. Matamoros, Tamaulipas, México.

\begin{tabular}{|c|c|c|c|c|c|c|}
\hline \multirow{2}{*}{ Variables } & \multirow{2}{*}{ Media } & \multirow{2}{*}{$D E$} & \multicolumn{2}{|c|}{ IC 95\% } & \multirow{2}{*}{$F$} & \multirow[b]{2}{*}{$p$} \\
\hline & & & LI & LS & & \\
\hline \multicolumn{7}{|l|}{ Actitudes } \\
\hline Primero & 81.55 & 9.13 & 79.43 & 83.67 & & \\
\hline Segundo & 83.63 & 7.04 & 81.78 & 85.48 & 1.086 & .340 \\
\hline Tercero & 82.90 & 8.39 & 80.98 & 84.82 & & \\
\hline \multicolumn{7}{|c|}{ Actitud Preventiva } \\
\hline Primero & 32.64 & 5.38 & 31.40 & 33.89 & & \\
\hline Segundo & 33.58 & 5.23 & 32.20 & 34.96 & .671 & .512 \\
\hline Tercero & 32.47 & 6.55 & 30.97 & 33.97 & & \\
\hline \multicolumn{7}{|c|}{ Actitud Permisiva } \\
\hline Primero & 11.62 & 4.20 & 10.64 & 12.59 & & \\
\hline Segundo & 11.41 & 3.67 & 10.44 & 12.37 & 6.296 & .002 \\
\hline Tercero & 13.53 & 3.88 & 12.65 & 14.42 & & \\
\hline \multicolumn{7}{|c|}{ Consecuencias Negativas } \\
\hline Primero & 18.04 & 3.59 & 17.57 & 19.23 & & \\
\hline Segundo & 19.65 & 2.26 & 19.05 & 20.25 & 8.063 & $<.001$ \\
\hline Tercero & 20.26 & 2.46 & 19.69 & 20.82 & & \\
\hline \multicolumn{7}{|c|}{ Facilitación Social } \\
\hline Primero & 7.51 & 2.44 & 6.94 & 8.08 & & \\
\hline Segundo & 7.43 & 2.30 & 6.82 & 8.03 & .901 & .408 \\
\hline Tercero & 7.93 & 2.40 & 7.38 & 8.48 & & \\
\hline \multicolumn{7}{|c|}{ Actitud Evasiva } \\
\hline Primero & 8.82 & 1.67 & 8.43 & 9.21 & & \\
\hline Segundo & 8.70 & 1.96 & 8.19 & 9.22 & 17.648 & $<.001$ \\
\hline Tercero & 7.07 & 2.25 & 6.56 & 7.59 & & \\
\hline
\end{tabular}

Tabla 5 - HSD de Tukey para la actitud permisiva, consecuencias negativas y actitud evasiva por año escolar. Matamoros, Tamaulipas, México.

\begin{tabular}{|c|c|c|c|}
\hline Actitudes & (I) Grado & (J) Grado & $p$ \\
\hline \multirow[t]{6}{*}{ Actitud Permisiva } & Primero & Segundo & .952 \\
\hline & & Tercero & .009 \\
\hline & Segundo & Primero & .952 \\
\hline & & Tercero & .006 \\
\hline & Tercero & Primero & .009 \\
\hline & & Segundo & .006 \\
\hline \multirow[t]{6}{*}{ Consecuencias Negativas } & Primero & Segundo & .037 \\
\hline & & Tercero & .000 \\
\hline & Segundo & Primero & .037 \\
\hline & & Tercero & .447 \\
\hline & Tercero & Primero & $<.001$ \\
\hline & & Segundo & .447 \\
\hline \multirow[t]{6}{*}{ Actitud Evasiva } & Primero & Segundo & .939 \\
\hline & & Tercero & $<.001$ \\
\hline & Segundo & Primero & .939 \\
\hline & & Tercero & $<.001$ \\
\hline & Tercero & Primero & $<.001$ \\
\hline & & Segundo & $<.001$ \\
\hline
\end{tabular}




\section{DISCUSIÓN}

El promedio de todas las dimensiones resultó por arriba de la media, es decir una alta actitud preventiva, actitud permisiva, percepción de consecuencias negativas, actitud evasiva y facilitación social; para explicar dichos resultados se hizo un análisis para identificar diferencias de las actitudes ante el consumo de alcohol por género del adolescente, se observó que la actitud preventiva, actitud permisiva y facilitación social es diferente en mujeres en comparación con hombres, con mayor promedio en la puntuación en hombres. Las diferencias encontradas en las dimensiones de actitud permisiva y de facilitación social son similares a lo reportado en otro estudio realizado en población mexicana, ${ }^{8}$ sin embargo, respecto a la actitud preventiva, en este estudio se encontró mayor puntuación en mujeres contrario a lo reportado por Ocaña y cols. ${ }^{8}$

Las diferencias estadísticas encontradas en este estudio, en la actitud preventiva por género indican que es mayor en las mujeres, en comparación con los varones; este hallazgo puede explicarse en que, aunque en los últimos años se han presentado diversos cambios sociales y culturales, en el contexto mexicano el autocuidado y cuidado de otros recae en la mujer. Al respecto la literatura indica que las mujeres se juzgan a sí mismas por su manejo de las responsabilidades y por su habilidad para cuidar de otros y de sí mismas ${ }^{10}$, es decir, asumen de manera natural la prevención y búsqueda de la seguridad personal y de su contexto.

Otra situación que explica que las mujeres presenten mayor actitud preventiva es el tipo de crianza familiar, donde a la mujer se le permite sentir y expresar sus emociones con mayor libertad que en los varones, por lo tanto en ellas se cumple lo que refiere García y colaboradores ${ }^{11}$ que la familia funciona como amortiguador de los efectos de la adversidad, que facilita en sus integrantes el aprendizaje de estrategias de enfrentamiento, actitud hacia situaciones estresantes y vínculos que facilitan el apoyo en momentos de angustia. En el contexto de los sujetos de este estudio, la familia sigue siendo primordial, y la mujer cumple con roles sociales muy marcados, esto representa una importante variable para continuar investigando y así poder desarrollar nuevas propuestas en cuanto a las motivaciones en el actuar de hombres y 
mujeres, por lo que se sugiere integrar dichas variables en futuras investigaciones.

El presente estudio reflejó, además, que los varones poseen una actitud más permisiva que las mujeres, similar a lo encontrado en un estudio realizado en Chiapas $^{8}$, lo cual pudiera explicarse en que los hombres en la búsqueda de aceptación y necesidad de reconocimiento reflejan conductas que implican mayor riesgo, pues estas parecen darles mayor prestigio, lo cual es importante en esta etapa. En cuanto a las mujeres, ellas se sienten aceptadas de una manera diferente, pues el respeto lo ganan por la profundidad de sus relaciones, la responsabilidad en el cuidado de sí mismas y de los demás ${ }^{12}$.

En otro orden de ideas, los hombres resultaron con mayor facilitación social, lo que evidencia que a pesar de estar en frontera persiste la cultura tradicionalista en cuanto a costumbres y crianza. El hombre y la mujer son formados conforme al rol que "se debe de cumplir", en ellos persiste mayor libertad de experimentar, de arriesgarse $\mathrm{y}$ en algunos de los casos es en la misma familia donde se abren las puertas al consumo de sustancias psicoactivas como el alcohol ${ }^{11}$.
Al respecto, la teoría de los roles sociales indica que la cultura define lo masculino y femenino, por lo que se espera que el comportamiento de las personas sea congruente con los roles social y culturalmente definidos. Mientras que Rebollo-Catalán y otros ${ }^{12}$ plantean que, en el proceso de hacerse hombre, el adolescente emplea ciertos rituales y prácticas sociales para afirmar su masculinidad principalmente ante sus iguales. En el norte y noreste de México, es común que en las reuniones familiares y sociales se prepare "carne asada", en el proceso de preparación la familia y amigos consumen bebidas alcohólicas, donde el adolescente puede tener acceso a ellas.

Por otra parte, se hizo un análisis para identificar la relación entre la actitud ante el consumo de alcohol y la edad, donde se identificó que, a mayor edad, mayor actitud permisiva y mayor percepción de consecuencias negativas y a mayor edad menor actitud evasiva. El mismo dato se encontró al analizar la diferencia de la actitud ante el consumo de alcohol por año escolar, donde se observó que la actitud permisiva, percepción de consecuencias negativas y la actitud evasiva fue diferente de acuerdo con el año escolar. 
La relación encontrada entre la actitud permisiva y la edad explica lo ya documentado en la literatura donde se indica que los jóvenes de mayor edad "aparentemente" muestran cierta madurez en muchos aspectos, ya que, el desarrollo del cerebro inmaduro puede permitir sentimientos que anulan la razón e impiden que presten atención a las advertencias que los adultos consideran lógicas y persuasivas. En este sentido, Papalia y colaboradores ${ }^{10}$ refieren que la toma de riesgos resulta de la interacción de dos redes cerebrales: 1) socioemocional y 2) red de control cognoscitivo que regula las respuestas a los estímulos. La red socioemocional incrementa su actividad en la pubertad, mientras que la red del control cognoscitivo madura de manera más gradual hacia la adultez temprana.

Sin embargo, aunque los adolescentes de mayor edad presentaron mayor actitud permisiva, también resultaron con mayor percepción de consecuencias negativas, lo que sugiere que en el contexto actual, los jóvenes están informados, tienen conocimientos sobre las consecuencias de consumo de alcohol y algunos de ellos tienen experiencias cercanas sobre los efectos a corto, mediano y largo plazo, fruto del consumo de alcohol o cualquier otra sustancia psicoactiva; sin embargo, estas variables no fueron medidas en este estudio, por lo que se sugiere profundizar en ellas en futuras investigaciones, ya que los adolescentes igual que los adultos, con el funcionamiento de los lóbulos frontales ejecutan la planeación, razonamiento, juicio, regulación emocional y control de impulsos, lo cual permite hacer juicios razonados más certeros ${ }^{10}$, en este caso, la percepción de las consecuencias del consumo de alcohol.

Así mismo, se encontró que a mayor edad menor actitud evasiva, lo que indica que, aunque los jóvenes tienen conocimiento de las consecuencias, esto no es garantía para tener actitudes negativas hacia el consumo de alcohol. Papalia y colaboradores $^{10}$ afirman que los hallazgos sobre la red socioemocional pueden ayudar a explicar por qué la toma de riesgos suele ocurrir en grupos, la tendencia de los adolescentes a mostrar arrebatos emocionales $y$ conductas de riesgo. Por otra parte, reportó que los adolescentes mayores (14 a 17 años) mostraron patrones más similares a los adultos (planeación, razonamiento, juicio, regulación emocional y control de impulsos, lo cual permite hacer juicios razonados más 
certeros). Esto parece confundir a los jóvenes en cuanto a la ilusión que se forman de poder sobre llevar las situaciones a las que se enfrenta, pues pareciera tener todo para ser asertivos, pero las decisiones muchas veces siguen siendo erróneas.

\section{CONCLUSIÓN}

Los resultados del presente estudio reflejaron una alta actitud preventiva, actitud permisiva, percepción de consecuencias negativas, actitud evasiva y facilitación social. La actitud preventiva, actitud permisiva y facilitación social es diferente en mujeres y hombres. A mayor edad, mayor actitud permisiva y mayor percepción de consecuencias negativas y a mayor edad, menor actitud evasiva.

Se reconoce que, por tratarse de adolescentes que estudian en una escuela privada, se limita la generalización de los resultados; sin embargo, se considera que es un importante acercamiento al estudio de las actitudes ante el consumo de alcohol en adolescentes; por lo que se sugiere continuar esta línea de investigación $e$ incluir estudiantes de instituciones públicas, bajo la premisa de que el factor socioeconómico pudiera ser una variable que influya en las actitudes.

\section{REFERENCIAS}

1. Secretaría de Salud. Aumenta el consumo de alcohol entre jóvenes. México; [Internet] 2016 [consultado el 27 de agosto de 2019]. Disponible en: https://www.gob.mx/salud/articulos/ aumenta-el-consumo-de-alcoholentre-jovenes

2. Organización Mundial de la Salud. Informe Mundial de Situación sobre Alcohol y Salud en las Américas. Washington, .C. [Internet] 2015 [consultado el 20 de marzo de 2020]. Disponible en: http:/ /iris.paho.org/xmlui/bitstream/ handle/123456789/7708/97892753185 53_esp.pdf

3. Trujillo Á, Flórez I. Consumo de Alcohol en los Adolescentes de Chía y su percepción del Consumo y de la Permisividad Parental frente al Uso de Sustancias. Rev Colomb Psicol. 2013; 22(1):41-57.

4. Armendáriz $N$, Alonso $M$, Alonso $B$, López M, Rodríguez L, Méndez M. La familia y el consumo de alcohol en estudiantes universitarios. Cienc Enferm. 2014; 20(3):109-118. 
5. Ruiz G, Medina-Mora M. La percepción de los adolescentes sobre el consumo de alcohol y su relación con la exposición a la oportunidad y la tentación al consumo de alcohol. Salud Mental. 2014; 37(1):1-8.

6. López-Cisneros MA, Villar MA, Alonso MM, Alonso MTJ, Rodríguez L. Actitud ante el consumo y no consumo de alcohol en estudiantes de preparatoria-México. Rev Esc Enferm USP. 2013; 47(4):815-821.

7. Pons D, Berjano P. El consumo abusivo de Alcohol en la adolescencia: Un Modelo explicativo desde la psicología social. Plan Nacional sobre Drogas. [Internet] 1999 [consultado el 15 de abril de 2020]. Disponible en: http://www.cedro.sld.cu/bibli/a/a4. pdf

8. Ocaña Z, García GA, Cruz Ó, Pérez CE. Actitudes hacia el consumo de alcohol en adolescentes chiapanecos. CNEIP. 2020; 2(1):84-96.

9. Secretaría de salud. Reglamento de la Ley general de Salud en Materia de
Investigación, México. [Internet] 1987 [consultado el 05 de febrero de 2020]. Disponible en: http://www.dof.gob.mx/nota_detalle .php?codigo $=5339162 \&$ fecha $=02 / 04 / 20$ 14

10. Papalia D, Feldman R, Martorell G. Desarrollo Humano. México: Editorial MCGRAW-HILL; 2017.

11. García J, García M, Rivera S. Potencial resiliente en familias con adolescentes que consumen y no consumen alcohol. Acta Colomb Psicol. 2015; 18(2):163-172.

12. Rebollo-Catalán Á, Ruiz-Pinto $E$, García-Pérez R. Preferencias relacionales en la adolescencia según el género. REDIE. 2017; 19(1):58-72.

Financiamento: Os autores declaram que não houve financiamento.

Conflito de interesses: Os autores declaram não haver conflito de interesses.

Participação dos autores:

- Concepção: Ontiveros-Patiño R, Sánchez GG, Aguilar MLLM, Ávila-Alpirez H, Guerra-Ordoñez JA, Duran-Badillo T.

- Desenvolvimento: Ontiveros-Patiño R, Sánchez GG, Aguilar MLLM, ÁvilaAlpirez H, Guerra-Ordoñez JA, Duran-Badillo T.

- Redação e revisão: Ontiveros-Patiño R, Sánchez GG, Aguilar MLLM, ÁvilaAlpirez H, Guerra-Ordoñez JA, Duran-Badillo T. 
Como citar este artigo: Ontiveros-Patiño R, Sánchez GG, Aguilar MLLM, Ávila-Alpirez H, Guerra-Ordoñez JA, Duran-Badillo T. Actitud ante el consumo de alcohol en adolescentes del Noreste de México. J Health NPEPS. 2021; 6(1):191-204.

Submissão: 03/10/2020

Aceito: $15 / 01 / 2021$

Publicado: 01/06/2021 